\title{
Design and Application of Vibrator Driven by Piezoelectric Stack Based on Low Frequency Vibration Cutting
}

\author{
KONG Fanxia \\ School of Agriculture and Food Engineering, Shandong University of Technology, Zibo City, \\ 255049, China \\ kfx0309@163.com
}

Keywords: Low-frequency vibration cutting; high-rigidity; piezoceramics; superalloys

Abstract: A new vibration cutting device was presented in the paper to offer much high power and high rigidity for the unstable vibration parameters, bad compliance between practical and theory which produced while using conventional vibration cutting equipment. Detailed calculation and structure design were carried out with special structure axis driven by piezoelectric stack which ensure output of amplitude to the real state. Confirmatory experiments were done and the experiment results showed that the new device achieves the true amplitude and the actual cutting performance which well matches the theoretical effect. This study provides a reliable basis for vibration cutting theory, experimental research and production.

\section{Introduction}

The vibration machining is an advanced manufacturing technology. For vibration cutting, the relationship of the relative motion changed between the tool and the workpiece as a certain vibration is added to the tool or workpiece. Vibration machining effectively solved some problems of hard cutting material machining and some precision cutting within a certain range. Excellent process effect showed in vibration cutting has been recognized by both academia and industry. [1-3] At the same time, theoretical studies of vibration cutting have been extensively conducted. [4-7] Currently, normal vibration generating device include mechanical eccentric vibration, solenoid / motor vibration, hydraulic vibration, the pneumatic vibration, magnetic vibration, piezoelectric vibration, and so on. [8-10] The above vibration generating device exist defects, such as poor process system rigidity in cutting processing, unstable process parameters, a large deviation between process parameters and theoretical parameters as the spring elastic recovery structure commonly used. For example, in vibration drilling process, chip-breaking effect will be able to produce theoretically as long as the amplitude selection satisfy the condition $A \geq f / 4$. However chip-breaking effect was not able to get until the amplitude selection satisfy the condition $\mathrm{A}=\mathrm{f}$ in some equipments. Relationship between the amplitude and the feed amount was always different in different equipment. The selected parameters need to be constantly adjusted to meet production requirements with the wear and tear of parts for conventional vibration generator. In short, it was seriously inconsistent with vibration cutting theory. The functional reliability of the vibration cutting device is very important for the effects of vibration cutting process and in-depth study of the vibration theory. A new vibration cutting device with piezoelectric actuator will be presented in this paper to provide a reliable basis for cutting theory, experimental research and production.

\section{Reliability design of the vibrator driven by piezoelectric}

Design the structure of the vibrators. Internal structure of the vibrator was designed based on the principle of real output amplitude and shown in Fig.1. The intermediate portion is a shaft with special structure, the form of the shaft is shown in Fig.2. Two groups of piezoelectric ceramic stack are played on the shaft shoulder spatial cross direction of $90^{\circ}$. The piezoelectric ceramic stacks were in the same length and the same area. The polarization is the same direction for the relative group piezoelectric ceramic and contrary direction for the two adjacent sets. When connected to a high voltage alternating 
current, the relative group piezoelectric ceramic will become longer while the adjacent sets become short. The elongation amount and the amount of shortening is equal and alternately.

Elongation and shortening promote the reciprocating motion of the intermediate shaft and give rise to the vibration of the shaft. Elongation or shortening of the ceramic stack is definitely the displacement amount of the intermediate shaft and is the axis amplitude as the interface between the ceramic stack end face and the shaft is a rigid contact surface. Reliability design of amplitude output is achieved in the vibration equipment with axis rigid reciprocating as elastic recovery agencies completely cancelled in this equipment.

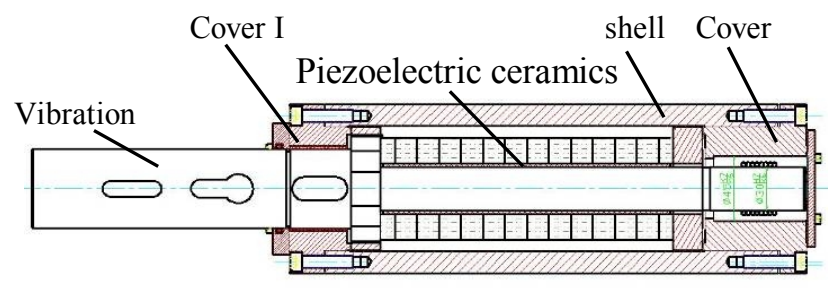

Fig.1 The vibrators structure schematic

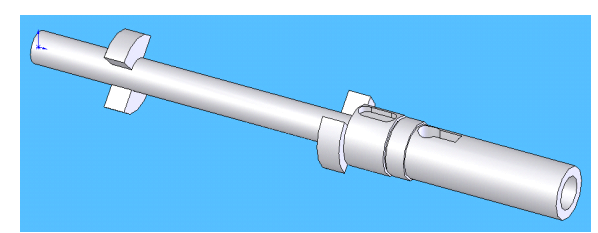

Fig.2 Diagram of the intermediate shaft

Design of ceramic reactor. Vibrator is designed to meet the conditions bellow. Material to be worked is high tensile steel, drilling aperture is ${ }^{\phi 20}$, feeding resistance is $10 \mathrm{KN}$, feed rate is $f=0.1 \mathrm{~mm} / \mathrm{r}$, amplitude is $A=40 \mu m$.

Firstly, determine the piezoelectric ceramic stack length and number of pieces. Piezoelectric ceramics PZT-8 is selected with piezoelectric constant $d_{33}=230 \times 10-12$, allowable voltage $\mathrm{Ua} \leq 1000 \mathrm{~V} / \mathrm{mm}$. Take the thickness of the piezoelectric ceramic $t=3 \mathrm{~mm}$. The number of piezoelectric ceramic sheet in the vibrator is derived by Eq.1. Finalized ceramic heap number of pieces is 60 . The total length is set to $200 \mathrm{~mm}$ combination of mechanical structures.

$$
m=\frac{A}{d_{33} U t}
$$

Then, determine the area of the piezoelectric ceramic. The energy provided by the piezoelectric ceramic stack should be able to meet the sum of energy consumption by the vibration member and cutting materials according law of conservation of energy. The maximum total power is determined by Equation 2) and calculated according vibration frequencies is $200 \mathrm{~Hz}, \mathrm{C}_{\mathrm{F}}=1800, \mathrm{~K}=1.5, \mathrm{M}=50 \mathrm{Mg}$, Angular frequency of vibration is $\omega$. Substituting a value of the design conditions $f 、 d_{0} 、 A_{\text {come into }}$ Eq.2, the power is calculated $1 \mathrm{Kw}$ and it is the power produced by the piezoelectric ceramic stack.

$$
P_{\max }=K\left(C_{F} d_{0} f^{0.7}+M A \omega^{2}\right) A \omega
$$

Every ceramic stack of piezoelectric ceramic is reduced to a capacitor with electric capacity $C$. Power of a capacitor circuit is $P=\omega C U^{2}$ according to the sinusoidal steady state. The electrical capacity of each ceramic sheets is gotten connected with the total power and the number of ceramic sheets pieces. The surface area of the ceramic sheet is identified according to the calculation formula of the plate capacitor Eq.3.

$$
C=\frac{\varepsilon A_{a}}{t}, \quad \varepsilon_{r}=\frac{\varepsilon_{33}}{\varepsilon_{0}}
$$

Wherein a capacitance is $\mathrm{C}(\mathrm{F})$, the electrode area is $A_{a}(\mathrm{~m} 2)$, the inter-electrode distance is $\mathrm{t}(\mathrm{m})$, permittivity is $\varepsilon(\mathrm{F} / \mathrm{m})$, relative permittivity is $\varepsilon_{r}$ (dimensionless).

Thus, the diameter of the annular ceramic sheet is gotten to $D=70 \mathrm{~mm}, d=35 \mathrm{~mm}$. The ceramic heap is designed as shown in Figure 3. The intermediate shaft is available to vibration with $50 \sim 200 \mathrm{~Hz}$ frequency and $0-40^{\mu m}$ amplitude. 


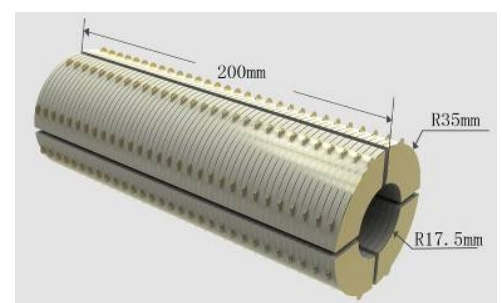

Fig.3 Piezoelectric ceramic stack

\section{Experiments and Results}

Measurement of amplitude. Amplitude output of vibrator corresponds to different amplitude voltage was measured using optical tool microscope. The relationship between the amplitude and the ceramic stack input voltage was shown in Figure 4. The measurement results show that amplitude grows linearly with the voltage increase, which is in line with the theory of piezoelectric relationship.

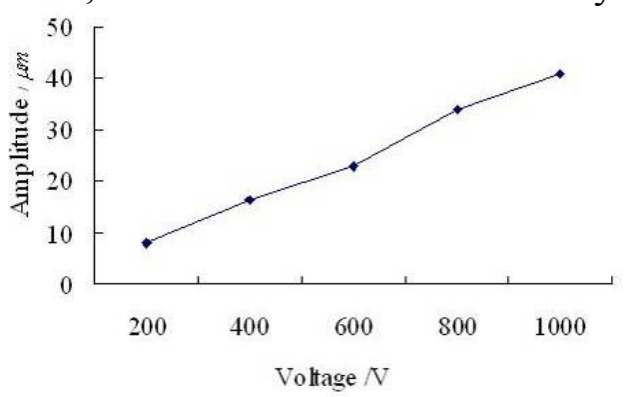

Fig.4 Amplitude voltage corresponding relationship

Drilling experiment of high temperature alloy for the verification. Metal drilling experiment were carried out respectively in the original mechanical vibration drilling system and the new piezoelectric ceramic driver vibration system to verify the stiffness and reliability of the system by comparing the chip breaking with the same cutting parameters and the same vibration parameters. Experimental equipment is shown in Figure 5.
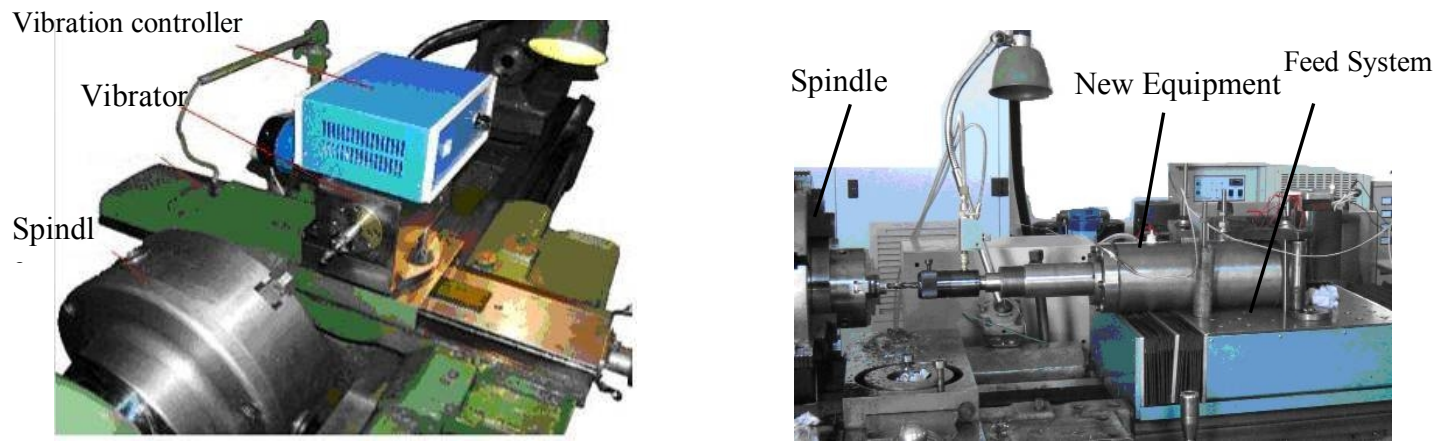

Figure 5 cutting experimental equipment

The parameters used in the experiments is: rotation speed is $400 \mathrm{r} / \mathrm{min}$, amount of feed is $0.025 \mathrm{~mm} / \mathrm{r}$, vibration frequency is $50 \mathrm{~Hz}$, amplitude were taken $0.006 \mathrm{~mm}, 0.013 \mathrm{~mm}, 0.020 \mathrm{~mm}$. A semi-synthetic cutting fluid emulsion and pouring cooling method was taken. Carbide drill tool diameter of $10 \mathrm{~mm}$ drill was used in high temperature alloy GH4169 which is one of embarrass processing materials. Chips were collected and experimental results were shown in Table.1. 


\begin{tabular}{|c|c|c|c|}
\hline $\begin{array}{l}\text { High rigid } \\
\text { vibration } \\
\text { equipment }\end{array}$ & 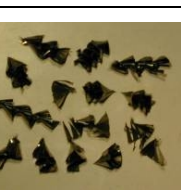 & 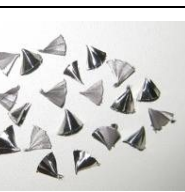 & 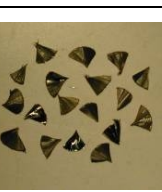 \\
\hline $\begin{array}{l}\text { Nomal vibration } \\
\text { equipment }\end{array}$ & & & \\
\hline amplitude $\mathrm{A}(\mathrm{mm})$ & 0.006 & 0.013 & 0.020 \\
\hline
\end{tabular}

Table.1 The state of the chip in two different equipments

The amplitude $\mathrm{A}$ is $0.006 \mathrm{~mm}$ (approximately equal $\mathrm{f} / 4$ ) if feed rate $\mathrm{f}$ is $0.025 \mathrm{~mm} / \mathrm{r}$ according the conditions chip break occurs based on the characteristics of separate chip breaking in vibration drilling. Experimental results showed that, the same as chip in ordinary drilling, there was not chip breaking effect on the chips collected from the mechanical vibration drilling system when the amplitude A takes $0.006 \mathrm{~mm}$ (approximately equal $\mathrm{f} / 4$ ). Chip breaking effect appeared when the amplitude take $0.013 \mathrm{~mm}$ (approximately equal $\mathrm{f} / 2$ ), but it does not satisfy the theoretical length. It will not have chip breaking effect consistent with theoretical until amplitude A is $0.02 \mathrm{~mm}$ (approximately equal $3 \mathrm{f} / 4$ ). While, chip breaking effect was good agreement with the theoretical when the amplitude A is $0.006 \mathrm{~mm}$ (approximately equal $\mathrm{f} / 4$ ) in vibration drilling system with high rigidity driven by piezoelectric ceramic. This gives a good example to prove that rigidity in the new system is higher than in machinery vibration drilling system. And the new system is suitable for cutting difficult to machine materials with the amplitude output stability and high rigidity.

Excellent vibration effects including amplitude reliable output and without distortion is provided by the new structure piezoelectric ceramic vibrator which excited with power supply. The new equipment can be used to machine difficult materials in drilling and reaming of large load rotational parts, especially to machine deep hole. The structure can also be used for non-rotational parts processing if using a rotary-powered.

\section{Conclusion}

1. New innovative equipment was proposed to achieve amplitude reliable output. Detailed process was given to design high-power high-rigidity vibrator driven by low-frequency piezoelectric ceramics.

2. The experimental results match with theoretical very well for chip breaking effect can occur when the amplitude is one fourth of the feed.

3. High-power high-rigidity vibration drilling technology is feasible after preliminary studies and provide a reliable basis for cutting theory, experimental research and production.

\section{Acknowledgments}

This work was partially supported by the National Natural Science Foundation (NSFC) Grant of China (NSFC-51205236).

\section{References}

[1] S.1 Arul, L.1 Vijayaraghavan, S.K.2 Malhotra, R.1 Krishnamurthy. International Journal of Machine Tools and Manufacture.2012, vol46(3-4):252-259

[2] Huafeng Shi, Runze Yang. Advanced Materials Research. 2013,Vol.630(2013):158-162

[3] Fanxia Kong, Deyuan Zhang. Journal of Beijing University of Aeronautics and Astronautics, 2012, vol 38(6), p 849-852 (in Chinese) 
[4] Fuqiang Tong, Feihu Zhang, Guangjun Chen,etal. Journal of Huazhong University of Science and Technology (Natural Science Edition). 2007,Vol.35(supp.1):68-70 (in Chinese)

[5] L.Z.1 Gu, Q Hong, C.J.Xiang. Key Engineering Materials. 2010,Vol426-427:320-324

[6] Yan Li, Lingfei Kong. Journal of Mechanical Engineering, v 48, n 13, p 167-173, July 5, 2012 (in Chinese)

[7] G.-L. Chern, J Liang. International Journal of Machine Tools and Manufacture, v 47, n 1, p 133-140, January 2007

[8] Lihua Xing, Xuchu Jiang, Deyuan Zhang, etal. ACTA ARMAMENTARII. 2007,28(6):739(in Chinese)

[9] Xueyong Chen, Zunrong Chen. Journal o f Fujian Agriculture and Forestry University (Natural Science Edition). 2010, Vol39(4) P431-434 (in Chinese)

[10] Lijie Ma, Guicheng Wang. Tool technology. 2009, Vol43(3):85-89 (in Chinese) 